\title{
Evaluation of Antifungal Efficacy of Cinnamomum zeylanicum bark oil
}

\section{Rashmi Mishra*, Shivansh Verma, Pratibha Pandey, Fahad Khan}

Department of Biotechnology, Noida Institute of Engineering and Technology, Greater Noida, India

Study Area: Greater Noida, India

Coordinates: $28.57^{\circ} \mathrm{N} ; 77.32^{\circ} \mathrm{E}$

Key words: Antifungal, Disease, Aromatic Oil, natural products

\section{Introduction:}

Increased interest in herbal medicine in the last twentieth century has provided an alternative perspective to the health care sector in developed as well as in developing countries. According to WHO estimates it was shown that about $80 \%$ of the world's population utilizing herbal medicine for their essential medical care because of its efficacy and lesser side effect (Prasansuklab \& Tencomnao, 2013). India possesses the gold-mine of traditional herbal medicine with well recorded and well-understood knowledge of herbal medicine. Ayurveda, one of the oldest existing medical systems is well recognized by $\mathrm{WHO}$ and is widely practiced. India recently has increased its research on traditional Ayurvedic herbal medicines after consideration that they are effective for the conditions to which they have been traditionally applied. Through literature review, itis evident that herbal medicine has a wide range of medicinal benefits and is gaining popularity all over the world probably because of lesser side effects (Ekor, 2013). Herb extract is used in liquid form, dried and powdered form directly in medicinal syrup, tinctures, and wines and in wide applications. Microorganisms such as fungi are found in air, soil, plants, and even on decayed organic material and are a major threat to human health causing acute and chronic human infections. Fungi affect a wide range including food crop disease such as smut disease in maize to food borne illness such as skin disease, digestive problems, diarrhea, vomiting, fever, abdominal cramps.

\section{Abstract}

Herbal medicine has a wide range of medicinal properties and the increasing use of herbal products in various applications by developed and developing countries has paved our way to curing diseases and conditions with natural herbs. Cinnamomum is an aromatic plant and its different species are widely studied. The aim of this study is to evaluate the antifungal efficacy of essential bark oil of Cinnamomum zeylanicum against the spectrum of pathogenic/non-pathogenic fungal cultures. The Hydrodistillation method was used for extracting essential oil from the bark of C.zeylanicum using Clevenger apparatus and the plate diffusion method was used to study the activity of tested fungi. The result obtained from this study suggests that $C$.zeylanicum essential bark oil could be a potential antifungal compound and thus may be useful in wide applications after evaluating the potential efficacy of essential oil for human use.

There is a small number of antifungal drugs available but major drawback includes the development of a high degree of resistance has been reported against the various strain of fungus. There is an urgent need for a possible alternative to involves the use of fungicides that are extracted from plant materials. 3 Secondary metabolites from medicinal plants and aromatic plants have widely used in perfume industries flavor and taste enhancer from ancient times (Anand et al., 2019; Vasconcelos et al., 2018). Cinnamomum plant is an aromatic plant and belongs to the family, Lauraceae and are reported to have biological properties such as analgesics, antiseptic, antioxidant astringent, carminative, antiviral, immunomodulatory, stimulant antitumor, and blood purifying properties and widely used from historic times by tribals in different ways all over the world (Wang et al., 2018; Ranasinghe et al., 2013). Polyphenols and volatile phenols are two main classes that are isolated from Cinnamomumzeylanicum (Nabavi et al., 2015).

Beneficial effects of C.zeylanicum have been shown in previous studies in-vivo as well as in-vitro (Mohamed et al., 2020; Ranasinghe et al., 2013). Several studies On Cinnamomum zeylanicum(native to Sri Lanka) utilizes the potential use of essential oils for the prevention and treatment of fungal disease and infection (Ranasinghe et al., 2002). Essential oil possesses the antibacterial, antifungal, and anti-plasmodial activity and have a pharmacological effect on various tissue and are currently being studied to procure a better understanding of their

*Corresponding Author: dr.mishra.rashmi@gmail.com 
RESEARCH ARTICLE

potential in natural food preservative (Vasconcelos et al., 2018; Condo et al., 2020; Wang et al., 2018; Mostafa et al., 2018; Ogunwande et al., 2003; Pandey et al., 2019; Arangannal et al., 2019). Studies demonstrated that essential oils affect the brain waves and they can alter the behavior as well (Condo et al., 2020). The previous study has examined the role of C.zeylanicum about a large number of bacteria (Wang et al., 2018; Mostafa et al., 2018; Ogunwande et al., 2003; Pandey et al., 2019; Arangannal et al., 2019; Mohamed et al., 2020; Ranasinghe et al., 2013). Keeping in view the ever-rising trend in herbal research, this study aimed to assess the antifungal activity of essential bark oil of Cinnamomum zeylanicum against seven mycotoxigenic fungi Aspergillus niger, Aspergillus flavus, Trichoderma viridae, Fusarium sp., Rhizopus stolonifer, Candida sp, Saccharomyces cerevisiae HAU-1, the study was conducted.

\section{Materials and methods:}

Preprocessing of plant sample: though every plant part possesses antibacterial and antifungal ability we selected the bark of C.zeylanicum for antifungal testing and was collected from the F.R.I botanical garden. The fresh plant samples were cleaned and washed with sterile distilled water and dried in shade for 4-6 days and crushed and weighed before calculating theyield.

Apparatus for Extraction of essential oils and their purification: in this study, the Clevenger apparatus was used for the extraction of essential oil. It is round bottom flask apparatus and consists of Clevenger main unit and condenser. A porcelain chip is added to a round bottom flask of Clevenger to avoid bumping of hot water. Extraction of essential oils is performed under atmospheric pressure at the boiling temperature of about $100^{\circ} \mathrm{C}$ (Hydrodistillation) after that oil starts coming within 10-15 min, and then heating mantle temperature is lowered between $65-70^{\circ} \mathrm{C}$ and this oil and water (emulsion) are collected in a conical flask and allowed to cool at room temperature. This oil is recovered by ether by using a separating funnel. Two phases are distinguishable, and this mixture of ether and oil was separated by filtration after 24hrand afterward, ether was evaporated on a water bath.

Preparation of culture media Potato Dextrose Agar (PDA) commercial media was used at a rate of $39 \mathrm{gm}$ in 100oml. pH was maintained to 4.8 and then sterilized at $1210 \mathrm{C}$ and $15 \mathrm{lbs}$ for $20 \mathrm{~min}$ in an autoclave.

Source of Test microorganisms: the test microorganism used in this study were: Aspergillus niger, Aspergillus flavus, Trichoderma viridae, Fusarium sp., Rhizopus stolonifer, Candida sp. All these microorganisms were obtained from departmental culture, and microorganism was subcultured on PDA culture media in different slants and stock culture was maintained at $4^{\circ} \mathrm{C}$.

Bore plate diffusion method: sterile agar plates of Potato Dextrose agar were prepared under aseptic conditions.
After solidification, 10oml of different microbial suspension was spread by spreader on each agar plate.2.6

Preparation of Microbial-suspension: the solution of different dilution concentrations of oil $(25 \%, 50 \%$, and $75 \%$ ) was prepared with $0.1 \%$ tween 20 (dissolved in sterile distilled water). Four wells were made on each agar plate on which organisms are already spread by borer of $8 \mathrm{~mm}$ diameter. Different dilutions of $25 \%, 50 \%$, and $75 \%$ of essential oil at a rate of $35 \mathrm{ml}$ were loaded at the center of each well. Plates were incubated at 25-280C for 7-8days. This assay was done in duplicate to ensure consistency.

Measurement of Inhibitionzone: after suitable incubation, the inhibition zone was measured in millimeters by using vernier calipers.

\section{Results:}

Antifungal activity of essential barkoils:

Inhibition assay of tested fungi with C.zeylanicum bark oil are shown in Table-1

Table-1. Mycelial growth inhibition assay with C.zeylanicum bark oil

\begin{tabular}{llll}
\hline Organisms & \multicolumn{3}{l}{ C.zeylanicum bark oil } \\
& $25 \%$ & $50 \%$ & $75 \%$ \\
\hline Aspergillus niger & 57 & 60 & 65 \\
Aspergillus flavus & 18 & 20 & 24 \\
Trichoderma viridae & 19 & 21 & 24 \\
Fusarium sp. & Plate clearance \\
Rhizhopus stolonifera & 22 & 45 & 50 \\
Saccharomyces cerevisai HAU-1 & 30 & 32 & 37 \\
Candida sp. & Plate clearance \\
\end{tabular}

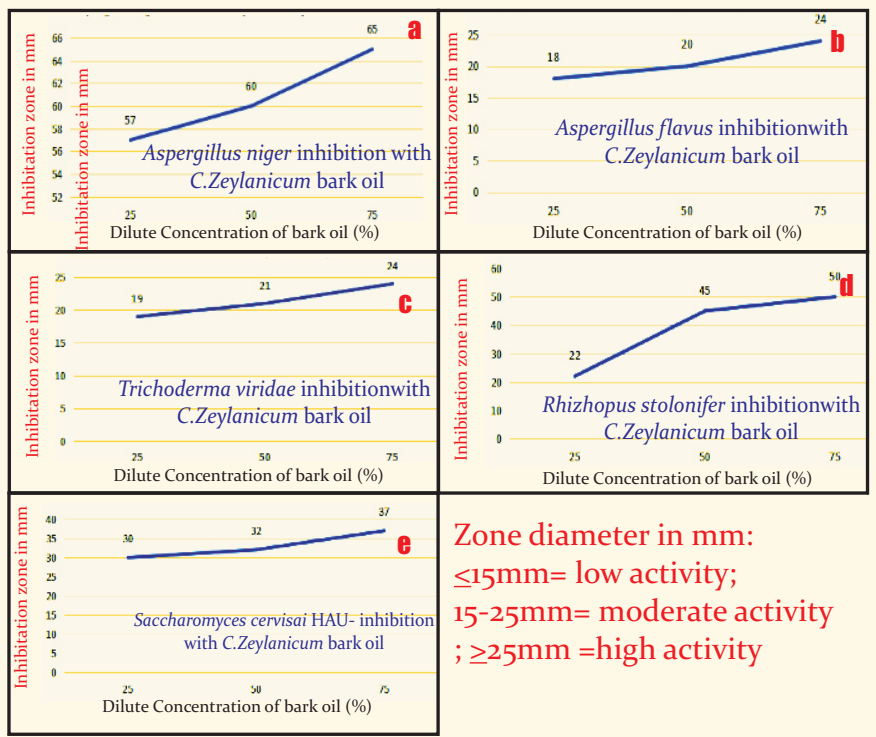

Figure 1: Mycelial Growth inhibition with $C$. zeylanicum bark oil (a) Aspergillus niger (b) Aspergillus flavus (c) Trichoderma viridae (d) Rhizopus stolonifer (e) Saccharomyces cervisai HAU-1 Fusarium sp. 
Effects of aromatic oil of C.zeylanicum on different organisms

Aspergillus niger: Bark oil of C.zeylanicum proved potent on culture of A.niger exhibiting inhibition zones ranged from $55 \mathrm{~mm}-65 \mathrm{~mm}$ (Table -1 ). Bark oil show its fungitoxic and fungicidal effect against A.niger (Fig.-1a).

Aspergillus Flavus: All concentration of C.zeylanicum bark oil exhibited moderate result at all concentrations against A.flavus,showing inhibition zones ranging from $18 \mathrm{~mm}-$ 24mm (Table-1 and Fig.-1b).

Trichoderma viridae: C.zeylanicum bark oil exihibited moderated result at all concentrations against Trichoderma viridae, exhibiting inhibition zone ranging from 19mm24mm (Table-1 and Fig.-1c).

Fusarium sp. : All concentration of C.zeylanicum bark oil showed over whelming response against Fusarium sp. Showing plate clearance which proved to be effective fungicidal against Fusarium sp.(Table-1).

Rhizopus stolonifer: Bark oil of C.zeylanicum showed potent result at all concentrations against Rhizopus stolonifer, producing a range of $20 \mathrm{~mm}-50 \mathrm{~mm}$ inhibition zone (Table-1 and Fig.-1d).

Saccharomyces cerevisae HAU-1: All concentration of C.zeylanicum bark oil exhibited inhibition zone ranging from 30mm-37mm against yeast (Table-1 and Fig.-1e).

Candida sp.: C.zeylanicum bark oil exhibited promising result against Candida sp. Giving total plate clearance result (Table-1).

\section{Discussion:}

Herbal medicine has been extensively used from traditional times having its applications in food preservation, alternative medicines, natural therapies, and pharmaceuticals. Increasing the incidence of infections by fungi and poor efficacy of drugs has to lead to draw our attention towards natural products as an antifungal (Ranasinghe et al., 2002; Nabavi et al., 2015). Previous studies have been reported for their antimicrobial properties (Wang et al., 2018). With the overuse of antibiotics increase in resistant bacterial and fungal strains has become a major cause for worldwide concern (Al-Bayati \& Mohammed, 2009). In a previous study, it was shown that in vivo studies indicate that cinnamon oil has very good curative effects in mice infected with Candida albicans (Wu \& $\mathrm{Li}$, 2013). Not much study has been reported for their antifungal efficacy of C.zeylanicum.This study gives an account of the antifungal activity of essential bark oil of C.zeylanicum. Essential oil is versatile and effective in treating many common problems (Saeed et al., 2018). Their antimicrobial effect of many herbs and spices has been known for centuries (Mohamed et al., 2020; Ranasinghe et al., 2013). C.zeylanicum has a high amount of cinnamaldehyde which is highly electronegative which interferes in biological processes that directly inhibit the growth of the microorganisms (Gupta et al., 2008). Several studies have been reported for their antifungal activities of essential oils of some Pakistani spices (Mostafa et al., 2018). The difference in their effects may be due to the number of phenolic compounds present in the essential oils and thus biological effects may directly depend on the type and quantity of active compounds (Chaudhari et al., 2012). The essential oil of leaves of C.tamala exhibited fungitoxicity against A.parasiticus (Pandey et al., 2019). Synthetic fungicides are used widely as a control method but they have developed drug resistance and thus causing a risk to health and the environment. Mycelial growth reduced upon increasing the concentration of essential oil. Therefore, further study should evaluate the new medicine and science to cure various diseases and conditions with better efficacy and lesser side effects.

\section{Conclusions:}

In conclusion, this study showed that the essential bark oil of C.zeylanicum possesses antifungal activity against tested microorganisms (Table-1). C.zeylanicum bark oil has shown an overwhelming response of plate clearance against Fusarium sp. and Candida sp. This study could guide the research on the effect of essential oil on different microorganisms and could be used in wide applications after evaluating the potential safety and efficacy of this essential bark oil for human health. There is a need for an hour to find out more natural products and screen them for further pharmacological studies that could pave the way for finding the new clinically effective antifungal compounds. Further, research is required to determine the mode of action of essential bark oil against these fungi and their effect on a large scale.

\section{References:}

Al-Bayati, F.A. \& Mohammed, M.J. (2009): Isolation, identification, and purification of cinnamaldehyde from Cinnamomum zeylanicum bark oil. An antibacterial study. Pharmaceutic. Biol., 47(1):61-6.

Anand, U., Jacobo-Herrera, N., Altemimi, A. \& Lakhssassi, N. (2019): A comprehensive review on medicinal plants as antimicrobial therapeutics: potential avenues of biocompatible drug discovery. Metabolites, 9(11):258.

Arangannal, P., Nithya, S., Jeevarathan, J., Rekha, V., Krishnan, M. \& Padmavathy, K. (2019): Antibacterial effectiveness of cinnamon chewing gum on Streptococcus mutans. Int. J. Public Health Res. E Develop., 10(8):1694-1698.

Chaudhari, L.K., Jawale, B.A., Sharma, S., Sharma, H., Kumar, C.D. \& Kulkarni, P.A. (2012): Antimicrobial activity of commercially available essential oils against Streptococcus mutans. L. Contemp. Dent. Pract., 13(1):71-74.

Condò, C., Anacarso, I., Sabia, C., Iseppi, R., Anfelli, I., Forti, L., de Niederhausern, S., Bondi, M. \& Messi, P. (2020): Antimicrobial activity of spices essential oils and its effectiveness on mature biofilms of human pathogens. Natural Product Res., 34(4):567-74. 


\section{RESEARCH ARTICLE}

Ekor, M. (2013): The growing use of herbal medicines: issues relating to adverse reactions and challenges in monitoring safety. Front Pharmacol., 4:177.

Gupta, C., Garg, A.P., Uniyal, R.C. \& Kumari, A. (2008): Comparative analysis of the antimicrobial activity of cinnamon oil and cinnamon extract on some food-borne microbes. Af. J. Microbiol. Res., 2(9):247-51.

Kaskatepe, B., Kiymaci, M.E., Suzuk, S., Erdem, S.A., Cesur, S. \& Yildiz, S. (2016): Antibacterial effects of cinnamon oil against carbapenem-resistant nosocomial Acinetobacter baumannii and Pseudomonas aeruginosa isolates. Indust. Crops Prod., 81:191-194.

Mohamed, A.E., Abdur, R. \& MM, S.A. (2020): Cinnamon bark as antibacterial agent: A mini-review. GSC Biol. Pharmaceut. Sci., 10(1):103-108.

Mostafa, A.A., Al-Askar, A.A., Almaary, K.S., Dawoud, T.M., Sholkamy, E.N. \& Bakri, M.M. (2018): Antimicrobial activity of some plant extracts against bacterial strains causing food poisoning diseases. SaudiJ. Biol.Sci., 25(2):361-366.

Nabavi, S.F., Di Lorenzo, A., Izadi, M., Sobarzo-Sánchez, E., Daglia, M. Nabavi, S.M. (2015): Antibacterial effects of cinnamon: From farm to food, cosmetic and pharmaceutical industries. Nutrients, 7(9):7729-7748.

Ogunwande, I.A., Olawore, N.O., Adeleke, K.A. \& Ekundayo, O. (2003): Chemical composition of essential oil of myristica fragrans houtt (nutmeg) from nigeria. J. Essen. Oil Bearing Plants, 6(1):21-26.

Pandey, P., Bajpai, P., Siddiqui, M.H., Sayyed, U., Tiwari, R., Shekh, R., Mishra, K. \& Kapoor, V.K. (2019): Elucidation of the chemopreventive role of stigmasterol against Jabı in Gall bladder carcinoma. Endocr. Metab. Imm. Disord. Drug Target., 19(6):826-837.
Ambient Science, 2021: Vol. 08(1); 24-27 DOI:10.21276/ambi.2021.08.1.ra04

Prasansuklab, A. \& Tencomnao, T. (2013): Amyloidosis in Alzheimer's disease: the toxicity of amyloid-beta (Aß), mechanisms of its accumulation and implications of medicinal plants for therapy. Evidence-Based Complement. Alternat. Med., 2013(3):413808

Ranasinghe, L., Jayawardena, B. \& Abeywickrama, K. (2002): Fungicidal activity of essential oils of Cinnamomum zeylanicum (L.) and Syzygium aromaticum (L.) Merr et LM Perry against crown rot and anthracnose pathogens isolated from banana. Lett. Appl. Microbiol., 35(3):208-211.

Ranasinghe, P., Pigera, S., Premakumara, G.S., Galappaththy, P., Constantine, G.R. \& Katulanda, P. (2013): Medicinal properties of 'true'cinnamon (Cinnamomum zeylanicum): a systematic review. BMC Complement. Alternat. Med., 13(1):275.

Saeed, M., Kamboh, A.A., Syed, S.F., Babazadeh, D., Suheryani, I., Shah, Q.A., Umar, M., Kakar, I., Naveed, M., ABD El- Hack, M.E., Alagawany, M. \& Chao, S. (2018): Phytochemistry and beneficial impacts of cinnamon (Cinnamomum zeylanicum) as a dietary supplement in poultry diets. World Poult. Sci. I., 74(2):331-346.

Vasconcelos, N.G., Croda, J. \& Simionatto, S. (2018): Antibacterial mechanisms of cinnamon and its constituents: A review. Microbial Pathogen., 120:198-203.

Wang, Y., Zhang, Y., Shi, Y.Q., Pan, X.H., Lu, Y.H. \& Cao, P. (2018): Antibacterial effects of cinnamon (Cinnamomum zeylanicum) bark essential oil on Porphyromonas gingivalis. Microbial Pathogen., 116:26-32.

Wu, Y. \& Li, M. (2013): Induction of tetraploid plants of Pogostemon cablin (Blanco) and its quality evaluation. Pharmacognosy J., 5(6):281-5. 